\title{
Sensor selection for fault diagnostics using performance metric
}

\author{
J. Reeves, R. Remenyte-Prescott, J. Andrews \\ Resilience Engineering Research Group, University Of Nottingham, University Park, \\ Nottingham, NG7 2RD, UK
}

Corresponding author: R. Remenyte-Prescott: R.Remenyte-Prescott@ nottingham.ac.uk

\begin{abstract}
$\underline{\text { Abstract }}$
As technology advances, modern systems are becoming increasingly complex, consisting of large numbers of components, and therefore large numbers of potential component failures. These component failures can result in reduced system performance, or even system failure. The system performance can be monitored using sensors, which can help to detect faults and diagnose failures present in the system. However, sensors increase the weight and cost of the system, and therefore, the number of sensors may be limited, and only the sensors that provide the most useful system information should be selected.

In this paper, a novel sensor performance metric is introduced. This performance metric is used in a sensor selection process, where the sensors are chosen based on their ability to detect faults and diagnose failures of components, as well as the effect the component failures have on system performance. The proposed performance metric is a suitable solution for the selection of sensors for fault diagnostics. In order to model the outputs that would be measured by the sensors, a Bayesian Belief Network (BBN) is developed. Sensors are selected using the performance metric, and sensor readings can be introduced in the BBN. The results of the BBN can then be used to rank the component failures in order of likelihood of causing the sensor readings. To illustrate the proposed approach, a simple flow system is used in this paper.
\end{abstract}

Keywords: Sensor selection; Bayesian Belief Network; Fault diagnostics;

\section{Introduction}

Aircraft systems, such as fuel systems, can be very complex, since they typically consist of a large number of different components, each of which can fail, sometimes in more than one failure mode. A failure of individual components and their groups can have different effects on the performance of the system, i.e. some failures may affect system performance severely and even cause system failure, but others may have very little effect. In order to be able to monitor system performance and detect early signs of system failure, sensors can be used on the system. These sensors can be all of one type, or a combination of multiple different types, such as flow, pressure and level sensors on a fuel system. In addition, if the sensors can be used to diagnose a component failure, a required action can 
be undertaken accordingly. For aircraft systems, this could be to abort the mission if it is unsafe for the aircraft to continue, adapt the mission if the aircraft is still safe to operate, but unable to complete the originally planned mission, or to continue the mission as normal if the component failures have no detrimental effect on the operation of the aircraft. If the mission does not have to be aborted, knowing component failures can help to plan the required maintenance work to be undertaken at the next scheduled stop, therefore reducing the unplanned down-time for the aircraft.

However, whilst it is desirable to position as many sensors as possible in order to have as much information as possible, it is not always practical. Sensors have associated costs, such as purchase, installation and the maintenance cost. All these costs increase the cost of building and operating an aircraft. In addition, in weight critical systems, such as aircrafts, including a large number of sensors can be infeasible. It is possible that some additional sensors do not contribute any additional information about the system behaviour, or some sensors might not be reliable and a level of redundancy to the sensor system is needed. Therefore, a balance between the number of sensors and their position on the system needs to be achieved, so that suitable information about the system performance is obtained without increasing the weight and the cost of the system unnecessarily.

A number of different approaches for selecting the best sensor combination for various different types of systems have been developed in literature. One way is to select the sensors which detect the most component failures. Snooke ${ }^{1}$ and Kang \& Golay ${ }^{2}$ developed fault symptom matrices in order to determine suitable sensor combinations in terms of their ability to detect failures. Each column in a fault symptom matrix corresponds to a different component failure, and each row corresponds to a different sensor. The matrix is then completed using " 1 " or " 0 " to represent whether the sensor can detect the component failure or not, respectively. The combination of sensors that can detect the most component failures can then be selected. The likelihood of component failure is not considered in this approach, i.e. sensors are selected according to their ability to detect component failures, irrespectively of their failure probability.

Authors, such as Memik et al. ${ }^{3}$ and Mukherjee et al. ${ }^{4}$, aimed to optimise the location of thermal sensors in microprocessors. Memik et al. ${ }^{3}$ state that there are typically two methods for distributing sensors in such systems: uniform sensors are distributed in a grid like pattern, and non-uniform sensors have an increased concentration of sensors in sections of the system that have more hotspots. However, the authors state that the location of hotspots can vary greatly depending on the application, and therefore requires significant testing to determine the most suitable locations. Despite this, Mukherjee et al. ${ }^{4}$ obtain a $75 \%$ reduction in the number of required sensors by distributing the sensors non-uniformly, using an algorithm to consider the number of hotspots within each potential sensor coverage area. The usage of such approached demonstrates the benefit of considering sensor locations with higher importance.

A performance metric for aircraft systems was developed by Maul et al. ${ }^{5}$, which was demonstrated using a subsystem of a space shuttle main engine. This method considers whether the sensors can detect enough of the failures, whether the sensor selection can discriminate between the component failures in order to be able to diagnose them and whether the sensor selection can account for sensor failures. However, as this suggested method requires some human input for some of the model parameters, the suggested performance metric, and therefore the sensor selection process, can be subjective. 
Khan \& Ceglarek ${ }^{6}$ developed a sensor optimisation process for fault diagnosis in multifixture assembly systems. The method consists of a sensor coverage index and a diagnosability index. However, like Maul et al..$^{5}$, the diagnosability index includes a weighting factor which represents the relative importance of different fault types. This requires the importance of each type of fault to be determined, and may introduce some subjectivity into the method.

Pourali \& Mosleh ${ }^{7}$ developed a utility function in order to select the best set of sensors. Expected values of the utility functions represent a decision maker's preference on a monetary scale, i.e. the higher the value, the better the sensor. This work outlines an algorithm for modelling the system using a BBN and selecting the best combination of sensors for the system using the utility function. The ability of the sensor selection to distinguish between different combinations of component failures, i.e. diagnose failures, is not considered.

Lambert \& Farrington ${ }^{8}$ used a cost-benefit function in order to select the best sensor combination, for the detection of chemical, biological and radiological air contaminants. The work considers several case-specific factors, including the density of elderly people, the predicted cost of treatment per affected person and the travel time to safety for the affected people. Many of these factors are case-specific, and the method appears to be unsuitable to other types of systems.

A number of authors, such as Santi et al. ${ }^{9}$, Spanache et al. ${ }^{10}$, and Maul et al.., ${ }^{5}$ used genetic algorithms to optimise the sensor selection process for various systems, including aircraft/spacecraft and a benchmark actuator. This enables a large number of different sensor combinations to be considered, without evaluating all possible combinations, especially avoiding those that have low fitness function value, used as the sensor selection criterion.

Other authors, including Rosich et al. ${ }^{11}$, Sarrate et al. ${ }^{12}$ and Krysander \& Frisk ${ }^{13}$, used structural methods for determining sensor combinations. In these methods, a system model is constructed which also includes possible sensor locations. The most suitable combination of sensors can be determined by using an algorithm for the calculation of the selection metric. This method has been applied to a number of different systems, for example, Rosich et al. ${ }^{11}$ applied the method to a compressor fuel cell, Sarrate et al ${ }^{12}$ applied it to a water distribution network, and Krysander $\&$ Frisk $^{13}$ - to an industrial valve.

Fault tree analysis is one of the traditional system reliability assessment techniques, which has also been used as a model-based fault diagnostic technique. It is also regularly used system reliability assessment. Hurdle et al. ${ }^{14}$ used non-coherent-fault trees to model a simple water tank system. Non-coherent fault trees consider NOT logic, when working components states can contribute to the top event occurrence. The authors suggest that this method enables the system to be modelled more accurately, because some sets of symptoms can only be produced if some components are still working, and others are failed. The authors extend this methodology and apply it to an aircraft fuel system, Hurdle et al. ${ }^{15}$. A potential drawback of using fault trees for fault diagnostics is that an individual fault tree has to be developed for each possible set of symptoms. This means that there is a lot of fault tree development work required before the model can be used to diagnose failures.

An alternative method, digraphs, is used by Bartlett et al. ${ }^{16}$ to diagnose failures in the aircraft fuel system, also used by Hurdle et al. ${ }^{15}$. The digraph method is compared to the 
fault tree method, and the authors state that the fault tree method determines what combinations of component failures can cause each combination of sensor readings, whereas the digraph method enables the modelling of the effects of the failure propagating through the system. However, the main limitation of digraphs is that they require the strength of the relationship between events in the network to be classified. This adds a level of subjectivity to the method. The authors come to the conclusion that as the two techniques both have their positives and negatives, the two techniques should be used in combination.

Another modelling technique that can be used for fault diagnostics is Petri Nets. Lloyd et al. ${ }^{17}$ use Petri Nets to confirm whether the faults that are detected on an aircraft fuel rig are actual faults or are false arising's. The method considers whether the sensor readings produced by the system are within a certain tolerance of the predicted values, produced by the Petri Net. However, if the deviation in the sensor reading is relatively small, the failures may not be detected, but if the size of the tolerance is reduced, the number of false arisings will increase.

Bayesian Belief Networks (BBNs) is another system modelling technique that can be used to diagnose failures in the system. The BBNs can be built based on expert knowledge, or as suggested by Lampis \& Andrews ${ }^{18}$ built using the structure of fault trees. In the latter, the nodes in the network represent events in the fault tree. Evidence about a fault present in the system can be introduced into the $\mathrm{BBN}$ and it is used to calculate the probability of each of the components being failed, by considering the Conditional Probability Tables (CPTs) for each node in the network. This can then rank the likelihood of components of being failed. The BBN method is chosen as the most suitable system modelling and fault diagnostic technique in this study, as they are able to handle multiple failure modes for multiple components in the same model, and the probability of event occurrence can be obtained using evidence about system performance, provided by sensors.

In this paper, a novel sensor performance metric is proposed in order for it to be used in the sensor selection method. The performance metric takes into account the probability of detected component failures, the ease of diagnosing the component failure, and the effects that the detected component failures have on system performance. A novel feature of the proposed performance metric is the ability to consider each of the aspects (detection, diagnostics and failure criticality) individually, enabling the analyst to focus on each aspect as appropriate to their specific application. A BBN of the system can be created and can then be used to diagnose the component failures in the system. Sensor reading evidence will update the probability of each of the components being in the failed state, and the BBN will then be able to output the components that are most likely to have failed, and therefore the appropriate action could be undertaken accordingly.

In this paper, an example system is outlined in section 2 in order to be able to demonstrate the application of the method is given. Section 3 introduces the sensor performance metric and the methodology used for fault diagnostics. In section 4, the method is then applied to the example system. Section 5 discusses the application of the methodology to the example system, highlighting the areas of potential improvement for the methodology. In the final section, section 6 , the work presented in this paper is concluded and potential future work is outlined. 


\section{System Description}

An example system, shown in Figure 1 is introduced in this section, which is used to demonstrate the proposed methodology.

The system consists of 5 valves (V1, V2, V3, V4 and V5) and 2 pumps (P1 and P2). The flow in the system is from left to right, where it exits the system via a drain which is indicated by the down arrow on the right hand side of the system. 11 sensors could be positioned on the system, with sensors S1 - S10 positioned either side of each of the valves, and sensor S11 positioned before the drain. $\mathrm{N}_{\mathrm{s}}$ is the number of possible sensors, i.e. $\mathrm{N}_{\mathrm{s}}=$ 11 for this example system.

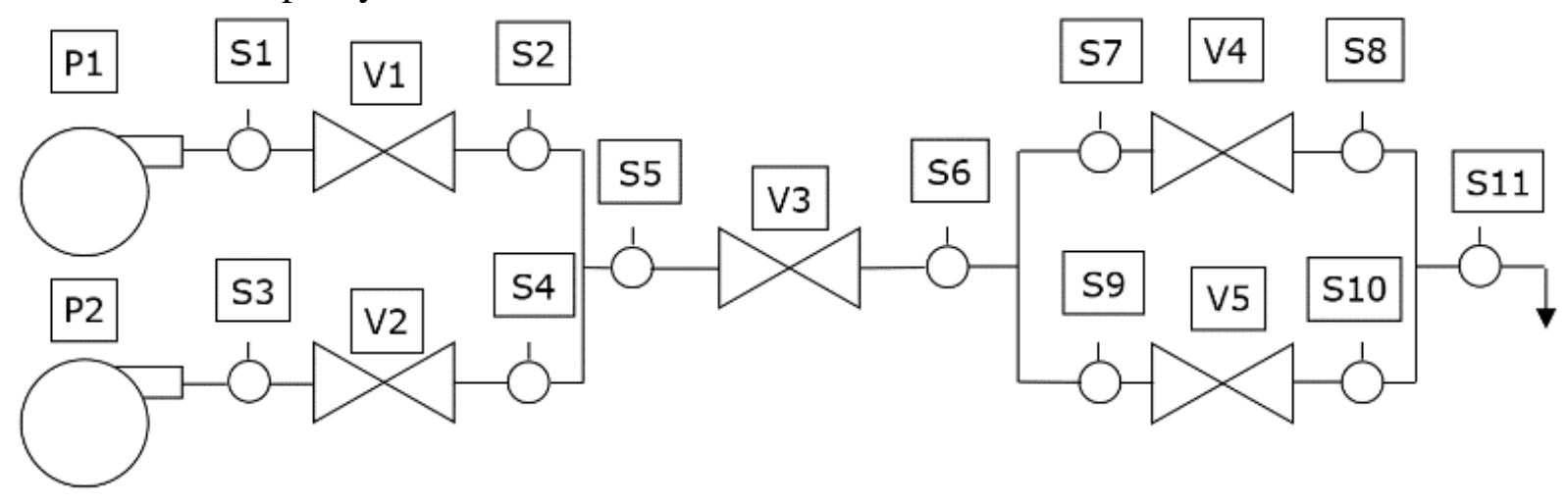

Figure 1 Example System

Under normal operating conditions, each of the pumps, P1 and P2, supply a quantity of fuel to the system, which passes through valve V1 and V2 respectively. This quantity is represented by "1" in Table 1. The fuel from the two parallel lines then combines and passes through valve V3. This quantity of fuel is represented by "2" in Table 1. The fuel then splits equally before going through the next two parallel lines with valves V4 and V5, at the same rate. This is at the same rate as supplied by each of the pumps and is represented by "1" in Table 1. Finally, the fuel combines again and leaves the system by passing through the drain. It is assumed that if there are two parallel lines, then the flow through each of the parallel lines is equal to half of the flow rate through the lines before and after the parallel section.

Each component in the system can be in one of two states: working or failed. The pumps are defined to be working when they are supplying fuel to the system. This is the fixed quantity of fuel, "1", as above. Only this quantity of supply is considered, along with no fuel supplied by the pump. The valves are defined to be working when they are allowing fuel to pass through the system unrestricted, and failed when they let no fuel pass through. No partial blockages of valves are considered in this system.

In this work, only combinations of one component failure and two component failures (occurring at the same time) are considered, i.e. three or more component failures are not considered, due to a very low probability of such events occurring. Note, for systems where the probability of combinations of three or more component failures have similar probabilities to combinations of one or two component failures, this assumption will not be valid. Therefore, in the proposed methodology, the combinations of one or two component failures are defined as the considered failures. An example of one component 
failing could be "pump P1 failed" and the rest of the components are working, and a combination of two components failing could be "pump P1 failed, and valve V1 failed" and the rest of the components are working. Note that it is assumed for the combinations of two component failures there is no time delay between the occurrences of the two failures.

Component failures can be critical to the performance of the system and they can even cause system failure. For this example system, system failure is defined as a failure that results in a reduction of the fuel exiting through the drain. This occurs when at least one of the pumps cannot supply fuel to the drain by a pump failing off, or by at least one valve being blocked, depending on the location of the valves. The minimal cut sets for this system failure logic are: $\{\mathrm{P} 1\},\{\mathrm{P} 2\},\{\mathrm{V} 1\},\{\mathrm{V} 2\},\{\mathrm{V} 3\}$ and $\{\mathrm{V} 4, \mathrm{~V} 5\}$. Note that if valve V4 or valve V5 (but not both) is blocked, there is no reduction in the supply of fuel from the pumps, since all the fuel can pass through the other line parallel to the one with the failed valve, and system failure does not occur.

In Table 1, the sensor readings for each combination of component failures are given. Note the first row of the table, case 0 , is when the system is working as normal, with no component failures. The sensor readings are as described above, with a "1" being the standard supply of fuel from a pump, " 2 " being double this value and " 0.5 " being half of this value. The final two sensor readings, "A" and "M", represent no flow of fuel through the lines with the lines being empty of fuel, and full of fuel, respectively. Note, " $A$ " and "M" are used instead of "0" in order to be able to distinguish between a situation with no fuel in the line, and hence no flow, and between a situation with fuel in the line, but no flow of fuel, respectively. In a real system, an interval of values might need to be considered in each category, for example, category " 1 " could be described by a range of values between 0.9 and 1.1, depending on the application.

Table 1 Sensor readings produced by failure combinations

\begin{tabular}{ccccccccccccc}
\hline No. & Failures & S1 & S2 & S3 & S4 & S5 & S6 & S7 & S8 & S9 & S10 & S11 \\
\hline 0 & No fault & 1 & 1 & 1 & 1 & 2 & 2 & 1 & 1 & 1 & 1 & 2 \\
1 & P1 & M & M & 1 & 1 & 1 & 1 & 0.5 & 0.5 & 0.5 & 0.5 & 1 \\
2 & P2 & 1 & 1 & M & M & 1 & 1 & 0.5 & 0.5 & 0.5 & 0.5 & 1 \\
3 & V1 & M & M & 1 & 1 & 1 & 1 & 0.5 & 0.5 & 0.5 & 0.5 & 1 \\
4 & V2 & 1 & 1 & M & M & 1 & 1 & 0.5 & 0.5 & 0.5 & 0.5 & 1 \\
5 & V3 & M & M & M & M & M & A & A & A & A & A & A \\
6 & V4 & 1 & 1 & 1 & 1 & 2 & 2 & M & M & 2 & 2 & 2 \\
7 & V5 & 1 & 1 & 1 & 1 & 2 & 2 & 2 & 2 & M & M & 2 \\
8 & P1 P2 & A & A & A & A & A & A & A & A & A & A & A \\
9 & P1 V1 & A & M & 1 & 1 & 1 & 1 & 0.5 & 0.5 & 0.5 & 0.5 & 1 \\
10 & P1 V2 & A & A & M & A & A & A & A & A & A & A & A \\
11 & P1 V3 & M & M & M & M & M & A & A & A & A & A & A \\
12 & P1 V4 & M & M & 1 & 1 & 1 & 1 & M & M & 1 & 1 & 1 \\
13 & P1 V5 & M & M & 1 & 1 & 1 & 1 & 1 & 1 & M & M & 1 \\
14 & P2 V1 & M & A & A & A & A & A & A & A & A & A & A \\
15 & P2 V2 & 1 & 1 & A & M & 1 & 1 & 0.5 & 0.5 & 0.5 & 0.5 & 1
\end{tabular}




\begin{tabular}{ccccccccccccc}
16 & P2 V3 & M & M & M & M & M & A & A & A & A & A & A \\
17 & P2 V4 & 1 & 1 & M & M & 1 & 1 & M & M & 1 & 1 & 1 \\
18 & P2 V5 & 1 & 1 & M & M & 1 & 1 & 1 & 1 & M & M & 1 \\
19 & V1 V2 & M & A & M & A & A & A & A & A & A & A & A \\
20 & V1 V3 & M & M & M & M & M & A & A & A & A & A & A \\
21 & V1 V4 & M & M & 1 & 1 & 1 & 1 & M & M & 1 & 1 & 1 \\
22 & V1 V5 & M & M & 1 & 1 & 1 & 1 & 1 & 1 & M & M & 1 \\
23 & V2 V3 & M & M & M & M & M & A & A & A & A & A & A \\
24 & V2 V4 & 1 & 1 & M & M & 1 & 1 & M & M & 1 & 1 & 1 \\
25 & V2 V5 & 1 & 1 & M & M & 1 & 1 & 1 & 1 & M & M & 1 \\
26 & V3 V4 & M & M & M & M & M & A & A & A & A & A & A \\
27 & V3 V5 & M & M & M & M & M & A & A & A & A & A & A \\
28 & V4 V5 & M & M & M & M & M & M & M & A & M & A & A \\
\hline
\end{tabular}

If as an example, case number 1 is considered, "pump P1 failed", then the only supply to the system will be from pump P2. Therefore, the fuel will pass through sensors S3 - S6 and they will each measure "1". The fuel will then split to flow equally through each of the parallel lines, resulting in sensors S7 - S10 measuring " 0.5 ". The fuel will then combine again and pass through sensor S11, which will measure "1". Sensors S1 and S2 measure "M", this is because there is no flow of fuel through these sensors as pump P1 has failed, however, there will be static fuel in the pipe from pump P2, hence the entry is "M" and not "A". The combinations of sensor readings in Table 1 can be used for the sensor selection process, as well as in the testing process of the fault diagnostic process, where sensor readings corresponding to a component failure can be introduced to the model, and the diagnosis of the failure can be verified accordingly. In the next section, the methodologies for sensor selection and fault diagnostics modelling are introduced.

\section{Proposed Methodology for Sensor Selection and Fault Diagnostics model}

The proposed methodology consists of two steps: the sensor selection, and the system modelling and fault diagnostics. The first step is to select the most suitable combination of sensors for the system. These sensors can then be included in the model of the system which is developed. This model can be used to diagnose failures in the system using the selected sensors. Example failures can be considered in order to demonstrate how effective the fault diagnostic process is.

\subsection{Performance metric methodology for sensor selection}

A novel sensor performance metric is proposed in this paper in order to select the most suitable sensors for this system. This considers the probability of the component failure occurrence which can be detected by the sensors, how easy it is to diagnose the failures, and what effect the component failures have on system performance, for example, how likely they are to cause the system to fail. For detecting faults, it is only required to be able to distinguish between the normal operating behaviour and the abnormal system behaviour produced when component failures are present. The fault diagnostic process aims to isolate 
the failures that have caused the abnormal system behaviour. The performance metric is limited to be between 0 and 1 , where 1 is the best performance value for the sensors, in order to have a clear reference for how good the sensors are. Sensor $s$ in the following equations for the performance metric terms refers to an individual sensor or a group of sensors.

\subsubsection{Detection Term}

In order for a fault to be detected by a sensor, the reading produced by the sensor must deviate from the reading produced under normal system behaviour. Note that for a group of sensors, only one of the sensors is required to have a deviated reading for the component failure to be detected, i.e. it is not necessary for all the sensors in the group to produce a deviated sensor reading.

$$
D E_{\{s\}}=\frac{P_{d}}{P_{m d}}
$$

In the detection term given in Equation $1, D E_{\{s\}}$, is equal to the ratio between the probability of occurrence of the detected failures and the probability of occurrence of the considered failures in the system. In Equation $1, P_{d}$ is the sum of probabilities of considered failures' occurrence that sensor $s$ can detect, and $P_{m d}$ is the sum of probabilities of considered failures' occurrence that can be detected by at least one sensor out of all the possible sensors on the system. Note that in some cases, there can be hidden failures that none of the sensors can detect, this would result in $P_{m d}$ being less than the sum of probabilities of considered failures occurrence. $D E_{\{s\}}$ is equal to 1 when sensor $s$ can detect all the considered failures that are possible to detect, and is equal to 0 when sensor $s$ cannot detect any of the failures that can occur on the system.

\subsubsection{Diagnostic Term}

The diagnostic term considers how easily a failure can be diagnosed using sensor $s$. For this term to be equal to 1, a unique sensor reading would need to be produced for every combination of component failures that can be detected. Note, that a combination of component failures can also refer to a single component failure.

However, in reality, the same sensor reading will be produced for multiple different component failure combinations, and therefore, the diagnostic term will normally be lower than 1 . The term will be close to 0 when there are many different component failure combinations that produce the same sensor reading and the failure combinations have similar occurrence probabilities. Having many different combinations of component failures with similar probabilities that produce the same sensor readings will reduce the likelihood of diagnosing the failures correctly.

The diagnostic term, $D I_{\{s\}}$, is shown in Equation 2. This term consists of two individual terms for each of the deviated sensor reading combinations that can be produced by sensor $s$. The first of these terms is $P_{s r i}$, which is the probability that a deviated reading $i$ of sensor $s$ occurs. For each of the possible combinations of deviated sensor readings, multiple different combinations of component failures can be their reason of occurrence. For each of these sensor readings, there will be a combination of component failures that will be the most likely to have caused the deviated sensor reading, therefore, $P_{m l i}$ is the probability of 
the most likely combination of component failures that can cause the reading $i$ of sensor $s$. These terms are summed over the number of different deviated readings of sensor $s, n r s$. Note, the sum of $P_{s r i}$ over all different deviated readings of sensor $s$ is equal to $P_{d}$, as introduced in section 3.1.1.

Therefore, $D I_{\{s\}}$ is the ratio between the sum of the probability of the most likely failure occurrence, and the sum of the probability for all the failure occurrence, across all the readings for sensor $s$. If there is more than one deviated sensor reading combinations that can be produced by sensor $s$, then the diagnostic term is equal to a weighted ratio of the probability of occurrence of the most likely component failure for each sensor reading and the probability of occurrence of all deviated sensor readings. This is weighted with respect to the probability of each of the deviated sensor reading combinations, with the deviated sensor reading combinations that are more likely to occur having a greater effect on the diagnostic term than the other terms. This is defined in the work, so that a sensor reading with a low occurrence probability, but the one that can only be caused by one combination of component failures, does not result in the diagnostic term being unrepresentative (overestimate) of the sensor performance.

$$
D I_{\{s\}}=\frac{\sum_{i=1}^{n r s} P_{m l i}}{\sum_{i=1}^{n r s} P_{s r i}}
$$

\subsubsection{Criticality Term}

The criticality term considers the effects that the component failures, detected by the sensors, have on the system performance.

This term is based on the Fussell-Vesely importance measure, Cheok et al. ${ }^{19}$. This importance measure was selected over other importance measures, such as, Birnbaum's importance measure and the criticality importance measure, because it could be adapted to consider sensors, and their groups with relative ease.

It considers the contribution of individual components (and their groups, as there is an equivalent measure for minimal cut sets) to system unavailability, i.e. the decrease in system unavailability when the component is made perfectly reliable in comparison to its failure. It is calculated by taking the probability of system failure and subtracting the probability that system failure occurs when component $j$ is working. The result is then normalised by the probability of system failure.

In order for this importance measure to be applied to sensors (instead of components), the subtracted term of the equation has been modified. This was changed to the probability of system failure, given that the non-deviated reading of sensor $s$ occurs.

The criticality term, $C R_{\{s\}}$, is given in Equation 3. In this equation, $Q_{s y s}$ is the probability of system failure with no additional knowledge on any of the components states, and $Q_{s y s}\left(q_{s}\right.$ $=0$ ) is the probability of system failure given that the non-deviated reading of sensor $s$ occurs.

$$
C R_{\{s\}}=\frac{Q_{s y s}-Q_{s y s}\left(q_{s}=0\right)}{\mathrm{Q}_{\text {sys }}}
$$


If sensor $s$ can detect all of the component failures, then $C R_{\{s\}}=1$. Alternatively, if sensor $s$ does not detect any of the critical component failures, then $C R_{\{s\}}=0$. In the following section, combining the three terms in order to form the sensor performance metric is discussed.

\subsubsection{Example}

In order to demonstrate the process of calculating the proposed performance metric, each of the three terms are calculated for sensor S1 of the system, presented in section 2 . According to Table 1, this sensor can detect 19 of the 28 component failures, 3 of which are single component failures and 16 are combinations of two component failures. It is assumed that the probability of each component failure, $A$, is 0.05 , such as $P(A)=0.05$. The probability that only one component is failed and all other components are working is represented as $P(A) \cdot(1-P(A))^{6}$, and the probability that only two components are failed and all other components are working is represented by $P(A)^{2} \cdot(1-P(A))^{5}$. Note, that the actual values of probabilities do not influence the ranking, i.e. only the ratio between the probabilities of different components needs to be realistic.

For sensor S1, $P_{d}$ is given in Equation 4, and $P_{m d}$ is given in Equation 5, respectively, and the detection term is therefore equal to $0.1412 / 0.2979=0.4740$.

$$
\begin{aligned}
& P_{d}=3 \times\left((0.05)(0.95)^{6}\right)+16 \times\left((0.05)^{2}(0.95)^{5}\right)=0.1412 \\
& P_{m d}=7 \times\left((0.05)(0.95)^{6}\right)+21 \times\left((0.05)^{2}(0.95)^{5}\right)=0.2979
\end{aligned}
$$

Sensor S1 produces two different deviated sensor readings, "M", and "A". The sensor reading " $\mathrm{M}$ " is produced by 16 of the 19 detected component failures ( 3 single component failures and 13 combinations of two component failures), and the sensor reading " $\mathrm{A}$ " is produced by 3 of the 19 detected component failures, all of which are combinations of two component failures. Therefore, $n r s$ is equal to 2 for sensor S1. $P_{m l i}$ for the sensor reading "M" and the sensor reading " $\mathrm{A}$ " are presented in Equations 6 and 7, respectively.

$$
\begin{aligned}
& P_{m l i}=(0.05)(0.95)^{6}=0.0368 \\
& P_{m l i}=(0.05)^{2}(0.95)^{5}=0.0019
\end{aligned}
$$

This is because the most likely failure for the sensor reading " $\mathrm{M}$ " is an individual component failure, and for the sensor reading " $A$ " - a combination of two component failures, respectively. $P_{s r i}$ for sensor reading "M" and sensor reading "A" are presented in Equations 8 and 9, respectively, which considers the number of combinations of single failures and two component failures.

$$
\begin{aligned}
& P_{s r i}=3 \times\left((0.05)(0.95)^{6}\right)+13 \times\left((0.05)^{2}(0.95)^{5}\right)=0.1354 \\
& P_{s r i}=3 \times\left((0.05)^{2}(0.95)^{5}\right)=0.0058
\end{aligned}
$$


Therefore, the diagnostic term for sensor S1 is equal to $(0.0368+0.0019) /(0.1354+0.0058)=0.2740$.

For the system, $Q_{s y s}$ is presented in Equation 10, since the only component failures that are not critical to system failure are V4 and V5, as stated in section 2. $Q_{s y s}\left(q_{s}=0\right)$ is given in Equation 11, i.e. it is the sum of the probabilities of component failures that cause system failure and that are not detected by sensor S1, divided by the sum of the probabilities of the occurrence of the non-deviated reading for sensor $\mathrm{S} 1$.

$$
\begin{aligned}
& Q_{\text {sys }}=5 \times\left((0.05)(0.95)^{6}\right)+21 \times\left((0.05)^{2}(0.95)^{5}\right)=0.2244 \\
& Q_{\text {sys }}\left(q_{s}=0\right)=\frac{2 \times\left((0.05)(0.95)^{6}\right)+5 \times\left((0.05)^{2}(0.95)^{5}\right)}{4 \times\left((0.05)(0.95)^{6}\right)+5 \times\left((0.05)^{2}(0.95)^{5}\right)+(0.95)^{7}}=0.0973
\end{aligned}
$$

Therefore, the criticality term is equal to $(0.2244-0.0973) / 0.2244=0.5665$.

\subsubsection{Discussion}

The performance metric consists of the three terms, described before. If each of the terms is equally important, then an average value of the three terms can be used as the performance metric, as shown in Equation 12.

$$
I_{\{s\}}=\frac{1}{3}\left(\left(\frac{P_{d}}{P_{m d}}\right)+\left(\frac{\sum_{i=1}^{n r s} P_{m l i}}{\sum_{i=1}^{n r s} P_{s r i}}\right)+\left(\frac{Q_{s y s}-Q_{s y s}\left(q_{s}=0\right)}{Q_{s y s}}\right)\right)
$$

However, the performance metric, expressed through this average value, can be unrepresentative of how useful the sensors are, if one of the terms is much greater than the other two terms. For example, if sensor $s$ only detects one component failure, then regardless of the probability of this component failure, the diagnostic term will be equal to 1. This would result in the performance metric being a significantly higher value than would be expected for a sensor that detects such a low percentage of component failures.

The performance metric could be affected in a similar way, if the probability of a critical failure is relatively low, in comparison to the probability of any failure occurring. If sensor $s$ detects all of the critical failures, but no others, this would result in a criticality term significantly higher than the detection term. As before, this would result in a higher performance metric than expected for a sensor that detects such a low percentage of component failures.

For most applications, it will be desirable to achieve a balance of all three terms, but there might be a need to favour one or two of the terms, at the expense of the other terms. A potential solution to this is to, first of all, use the average of the three terms to calculate the performance metric as suggested in Equation 12, but to then consider the terms individually for homing in the combinations of sensors with a high performance metric. This would result in a threshold being set that the performance metric needs to reach, but the combination of sensors that is most suitable to the particular application can be selected by looking at the individual terms. For example, the detection term of the performance 
metric could be favoured for low safety-risk systems, such as a car engine, where the car could pull over safely on the side of the road in the case of failure. The diagnostic term could be favoured for systems where system down-time is expensive, requiring the repair work to be completed as quickly as possible, such as a train engine, where the train would block the rail line, delaying other services. The criticality term could be favoured for safety-critical systems, where critical component failures need to be detected and mitigating actions need to be taken, such as emergency rerouting due to a failure of an aircraft engine. The proposed method allows such conditions to be satisfied, giving the analyst the ability to choose the best sensor combination for their particular application, a feature that is uncommon in previously published methods.

In the next section, the methodology for system modelling and fault diagnostics is introduced.

\section{2. $\quad$ Fault Diagnostics model}

In this section, the construction process of the fault diagnostic model is outlined. The fault diagnostics technique used in this paper is the BBN method. BBNs are probabilistic graphical models which are represented by directed acyclic graphs. Each network consists of a number of nodes, each of which has a finite number of discrete states. The probability of each of the states of the nodes are controlled by using Conditional Probability Tables (CPTs). The dependency of connected nodes is modelled using the CPTs, enabling the propagation of information through the system, Koski \& Noble ${ }^{20}$. The constructed BBN is used for the diagnostics of component failures in the system. The BBN in this paper was constructed using the software, "HUGIN Researcher".

When constructing a BBN for modelling the systems, the first step is to create a node for each of the components in the system. Each component node will have a state for each of the component states. For the example system, discussed in section 2, there are two states for each component node: working and failed. The next step in the construction process of the BBN is to introduce any intermediate nodes, if they are required. The intermediate nodes consider the state of groups of components and can be used to determine values of the parameters of the system, such as flow value at a junction. The final step is to include a node for each sensor. These nodes consider the states of the intermediate nodes in order to determine the sensor readings, with a state assigned to each possible sensor reading. Note, only the nodes for selected sensors need to be included in the network, however in this example all sensors were included in the network for completeness.

Intermediate nodes could be omitted from the network. However, if there are no intermediate nodes, the sensor nodes will have to consider more combinations of component states than if they were grouped together by the intermediate nodes. As the number of entries in the CPT for a node is equal to the product of the number of states for each of the parent nodes and the number of states in the node, the more states the parent nodes have, and the more parent nodes there are, and the larger the CPTs are for the child nodes. However, if no intermediate nodes are included, fewer nodes are required in the $\mathrm{BBN}$ to represent all the required information. The number of nodes in the network, and the size of the CPTs for the nodes should be balanced, so that the number of nodes in the network and the number of entries in the CPTs are not too high. 
Once the $\mathrm{BBN}$ is constructed, it can be used to diagnose component failures in the system. This is achieved by introducing sensor readings to the BBN as evidence. The state for each of the selected sensors that corresponds to the sensor readings is chosen, and the probability of the components being in each of their states is calculated. The probability of each state for each component can be calculated using Bayesian updating formulas, when evidence about system performance, captured by sensors, is introduced in the model. This step is completed automatically using the software, "HUGIN Researcher". The BBN will then identify which components are most likely to be in the failed state. If the identified component is not the component that has actually failed, then more evidence can be introduced, and the diagnosis is repeated. This additional evidence could be that a component is in its working state. The BBN not identifying the correct component failure initially is usually not due to a method limitation, it is due to multiple different component failures producing the same symptoms on the system, which is a common feature of systems.

In the next section, the proposed methodology is applied to the example system.

\section{Application of the Methodology}

As stated in Section 3.1.4, it is assumed that the probability of each component failure in the example system, $A$, is 0.05 , such as $P(A)=0.05$. Note, in a real system, component failure rates would be used as quoted by the manufacturer of the components.

\subsection{Sensor Selection}

The best possible performance metric can be calculated by considering all 11 sensors on the system at once. This process results in the performance metric value being equal to 0.8961: with detection and criticality terms of 1 , and a diagnostics term of 0.6883 . This means that it is possible to detect all of the considered combinations of component failures (as $D E_{\{s\}}=1$ ). As all considered component failures can also be detected, all critical failures can be detected $\left(C R_{\{s\}}=1\right)$. However, as the performance metric is less than 1 , and the value of $D I_{\{s\}}$ is less than 1, it suggests that the diagnostics approach based on these sensors does not diagnose all of the components correctly.

\subsubsection{Results}

This best possible performance metric, as discussed above, can be achieved by some combinations of four sensors. Therefore, there is no benefit to considering any more than four sensors. For illustration, the performance metric was calculated for all combinations of one, two, three and four sensors in this example. The rankings for individual sensors are given in Table 2, and the top six combinations of two and three sensors are given in Table 3. Note, in Table 3, only one combination of sensors is given per ranking for brevity. The reason that there are multiple combinations of sensors that have the same performance metric is that there is symmetry in the system because of the parallel fuel lines in the system. There are four combinations of four sensors that achieve the maximum performance metric, i.e. $I_{\{s\}}=0.8961, D E_{\{s\}}=1, D I_{\{s\}}=0.6883$, and $C R_{\{s\}}=1$. These combinations of sensors are:

- $\quad$ S1 S2 S3 S7

- S1 S2 S3 S9

- $\quad$ S1 S3 S4 S7 


\section{- $\quad$ S1 S3 S4 S9}

Table 2 Ranking of individual sensors for the system

\begin{tabular}{llllll}
\hline Rank & Sensor & $I_{\{s\}}$ & $D E_{\{s\}}$ & $D I_{\{s\}}$ & $C R_{\{s\}}$ \\
\hline 1 & S7 & 0.8062 & 0.9740 & 0.5067 & 0.9512 \\
& S8 & 0.8062 & 0.9740 & 0.5067 & 0.9512 \\
& S9 & 0.8062 & 0.9740 & 0.5067 & 0.9512 \\
2 & S10 & 0.8062 & 0.9740 & 0.5067 & 0.9512 \\
& S5 & 0.6688 & 0.7532 & 0.3362 & 1.0000 \\
3 & S6 & 0.6688 & 0.7532 & 0.3362 & 1.0000 \\
4 & S11 & 0.6667 & 0.7532 & 0.3276 & 1.0000 \\
& S1 & 0.3901 & 0.4740 & 0.2740 & 0.5665 \\
& S2 & 0.3901 & 0.4740 & 0.2740 & 0.5665 \\
& S3 & 0.3901 & 0.4740 & 0.2740 & 0.5665 \\
& S4 & 0.3901 & 0.4740 & 0.2740 & 0.5665 \\
\hline
\end{tabular}

Table 3 Ranking of combinations of 2 and 3 sensors for the system

\begin{tabular}{llllll}
\hline Rank & Sensors & $I_{\{s\}}$ & $D E_{\{s\}}$ & $D I_{\{s\}}$ & $C R_{\{s\}}$ \\
\hline 1 & S1 S7 & 0.8684 & 0.9870 & 0.6513 & 0.9754 \\
2 & S2 S7 & 0.8663 & 0.9870 & 0.6447 & 0.9754 \\
3 & S5 S7 & 0.8398 & 1.0000 & 0.5195 & 1.0000 \\
4 & S5 S8 & 0.8377 & 1.0000 & 0.5130 & 1.0000 \\
5 & S8 S10 & 0.8355 & 1.0000 & 0.5065 & 1.0000 \\
6 & S7 S8 & 0.8084 & 0.9740 & 0.5133 & 0.9512 \\
\hline 1 & S1 S3 S7 & 0.8939 & 1.0000 & 0.6818 & 1.0000 \\
2 & S1 S3 S8 & 0.8918 & 1.0000 & 0.6753 & 1.0000 \\
3 & S1 S4 S7 & 0.8896 & 1.0000 & 0.6688 & 1.0000 \\
4 & S1 S4 S8 & 0.8874 & 1.0000 & 0.6623 & 1.0000 \\
5 & S1 S8 S10 & 0.8853 & 1.0000 & 0.6558 & 1.0000 \\
6 & S2 S4 S8 & 0.8831 & 1.0000 & 0.6494 & 1.0000 \\
\hline
\end{tabular}

As can be seen by comparing the best combination of three sensors, there is very little benefit to considering an additional fourth sensor. As a result of this, and due to the large number of combinations of four sensors that are available (double the number of combinations of three sensors), further combinations of four sensors were not considered in this paper.

\subsubsection{Discussion}

If the individual terms of the performance metric in Table 2 are studied, it supports the suggestion of considering the three terms individually, rather than the average values, for the final sensor selection. As an example, sensors S7 to S10 are ranked the highest, but sensors S5 and S6 have a higher criticality term, even though they are ranked second. This 
would, most likely, lead to the analyst selecting either sensor S5 or S6, if it is imperative to be able to detect as many as possible of the critical failures.

Similar conclusions can be drawn when studying the combinations of two sensor rankings in Table 3. In this case, it can be seen that the sensor combination ranked $3^{\text {rd }}$ (S5 S7) detects all of the considered failures, and therefore has a detection term of 1 . The other sensor combinations that are ranked higher than this, such as (S1 S7), have a lower detection term. This will result in the analyst selecting the $3^{\text {rd }}$ best combination of sensors, if it is imperative to be able to detect all of the component failures.

When combinations of sensors consist of two sensors in close proximity to each other and located in the same section of the system, there is only a small increase in the performance metric, in comparison to a single sensor being positioned in that section. For example, the performance metric for the sensor combination (S7 S8) is 0.8084, but the performance metric for sensor S7 on its own is 0.8062 . In this case, only one of the terms has actually increased, i.e. the diagnostic term has changed from 0.5067 to 0.5133 . This results in the conclusion that it will normally be better to pick sensors that are not in close proximity to each other, as it is likely that the sensors that are in close proximity will provide similar information on what is happening in the system. This could be useful when the number of sensors needs to be minimised.

The maximum performance metric that is achievable for each different number of sensors increases for each additional sensor. However, for each additional sensor the returns are diminishing. This is in line with the motivation for finding a balance between the sensor cost and the quantity of information to be obtained.

For the fault diagnostics application on this system presented in the next section, the combination of sensors selected is: sensors S1, S3 and S7. This combination of sensors has the highest performance metric in Table 3. The position of these selected sensors on the system is such that there is a sensor positioned next to each pump and before the corresponding valve, and there is a sensor before one of the valves in the final section of the system. Using these three sensors, the flow throughout the entire system can be determined, since the sensors next to each of the pumps give information about fuel supply, and the sensor in the final section of the system informs the analyst about the fuel passing through the corresponding valve. The analyst can then determine the flow of fuel throughout the whole system, therefore, if there is any change in the system which results in a deviation from the flow under normal operating conditions, then these three sensors can detect it.

\subsection{Construction of the fault diagnostics model}

\subsubsection{BBN development}

As described in the proposed methodology, a node is added for each of the components in the BBN. The values in the CPTs correspond to the probability of each component being in the working or failed state, 0.95 and 0.05 respectively, as outlined above.

A number of intermediate nodes are introduced next, which are used to determine the flow at various points in the system. The intermediate nodes are used to split the example system into three sections. The first section consists of the two valves, V1 and V2, and the two pumps, P1 and P2. The second section consists of valve V3 only, and the final section consists of the remaining components, valves V4 and V5. Each of these nodes can be used 
to represent the supply of fuel downstream of the system, and also to indicate the lines that the fuel is able to pass through.

Finally, nodes are introduced to the network for each of the 11 considered sensors. These sensor nodes are connected to the intermediate nodes, and in some cases the component nodes, as required. Connecting the sensor nodes to the intermediate nodes means that the number of states that are required to be considered is reduced, in comparison to omitting the intermediate nodes. For example, for sensors S5 to S11, the only information required from section 1 of the system is the fuel supply. If it is " 1 ", it is unnecessary to know which pump the supply is coming from, and the reason why the fuel is not coming from the other pump (e.g. pump failed, valve failed). This results in fewer entries in the CPTs for each of the sensor nodes. For example, if the intermediate nodes in the first section are not included, then there will be 16 combinations of component states to consider for the components in the first section. However, including the intermediate nodes reduces the number of combinations down to 3, i.e. Full Supply (FS, "2"), Partial Supply (PS, "1") and No Supply (NS). This method therefore reduces the number of entries for each of the sensor nodes from 16 to 3 of the number of entries, if intermediate nodes are included.

The CPT for sensor S11 is given as an example in Table 4. This table demonstrates the reduction in size of the CPT. Without including the intermediate nodes in the second section included, for every three columns in Table 4, there would be 16 columns. The BBN of the system is given in Figure 2.

Table 4 CPT for sensor S11

\begin{tabular}{|c|c|c|c|c|c|c|c|c|c|c|c|c|c|c|c|c|c|c|c|c|c|c|c|c|}
\hline \multirow{3}{*}{$\begin{array}{l}\text { Section } 3 \\
\text { Valve } 3 \\
\text { Supply }\end{array}$} & \multicolumn{6}{|c|}{ Both Open } & \multicolumn{6}{|c|}{ V4 open, V5 blocked } & \multicolumn{6}{|c|}{ V5 open V4 blocked } & \multicolumn{6}{|c|}{ both blocked } \\
\hline & \multicolumn{3}{|c|}{ Open } & \multicolumn{3}{|c|}{ Blocked } & \multicolumn{3}{|c|}{ Open } & \multicolumn{3}{|c|}{ Blocked } & \multicolumn{3}{|c|}{ Open } & \multicolumn{3}{|c|}{ Blocked } & \multicolumn{3}{|c|}{ Open } & \multicolumn{3}{|c|}{ Blocked } \\
\hline & FS & PS & NS & FS & PS & NS & FS & PS & NS & FS & PS & NS & FS & PS & NS & FS & PS & NS & FS & PS & NS & FS & PS & NS \\
\hline No flow & 0 & 0 & 1 & 1 & 1 & 1 & 0 & 0 & 1 & 1 & 1 & 1 & 0 & 0 & 1 & 1 & 1 & 1 & 1 & 1 & 1 & 1 & 1 & 1 \\
\hline Half flow & 0 & 1 & 0 & 0 & 0 & 0 & 0 & 1 & 0 & 0 & 0 & 0 & 0 & 1 & 0 & 0 & 0 & 0 & 0 & 0 & 0 & 0 & 0 & 0 \\
\hline Normal flow & 1 & 0 & 0 & 0 & 0 & 0 & 1 & 0 & 0 & 0 & 0 & 0 & 1 & 0 & 0 & 0 & 0 & 0 & 0 & 0 & 0 & 0 & 0 & 0 \\
\hline
\end{tabular}

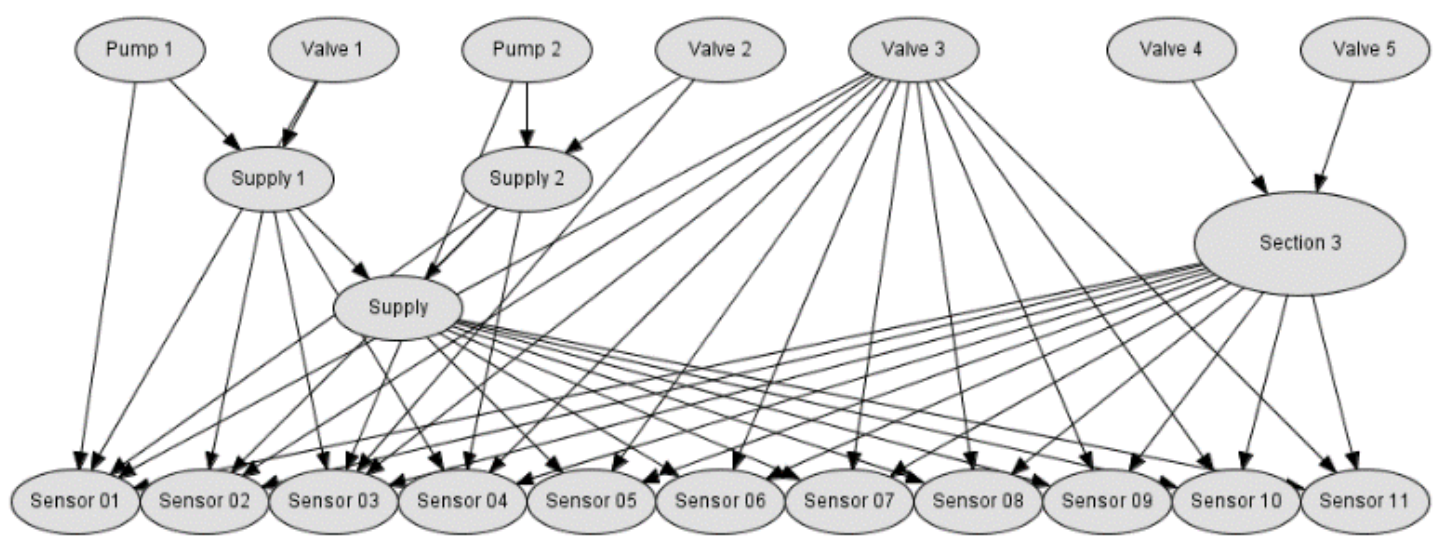

Figure 2 BBN of example system 


\subsubsection{Discussion}

The sensor nodes in the BBN are used to represent the sensors in the system. The node states represent the sensor readings that would be produced by the sensors on a system. Each state of the sensor nodes corresponds to a different sensor reading that can be produced by that sensor. If there are a large number of different potential sensor readings for a larger system, some of the sensor readings may have to be grouped together in ranges, in order to prevent the number of entries in the CPTs getting too large. However, this would result in a reduction of the accuracy of the model.

By introducing intermediate nodes to the network, the total number of entries in all of the CPTs is reduced. However, the process highlighted the fact that even with these intermediate nodes included, the total number of entries in all of the CPTs can be large, even for this simple example system with approximately 1400 entries in CPTs in the BBN. Alternatively, for large systems a structure learning algorithm could be used to construct the BBN automatically, removing the need to manually complete the CPTs. Mittal \& Maskara $^{21}$ present a review of some structure learning algorithms for BBN construction.

The BBN is used to aid in the diagnosis of failures in the system, as shown in the following section.

\subsection{Fault diagnostics}

The BBN fault diagnostics model is used to diagnose example component failures. The sensor combination selected is the sensors S1, S3 and S7, as outlined above. All of the combinations of component failures given in Table 1 are considered. The corresponding sensor readings for each of these combinations are introduced to the BBN as evidence, and the diagnostic process, as described in the final paragraph of section 3.2, is applied.

All of the component failures can be diagnosed using the BBN with relative ease. There were some cases that were not diagnosed correctly immediately, but no more than one additional component needed to be inspected for all the component failures to be diagnosed correctly. This demonstrates that the BBN can be used to diagnose failures in the system, using the selected sensors. In the following paragraphs, a number of example cases are outlined that demonstrate how successful the methodology is. The 28 different combinations of component failures are grouped in to seven different cases of different types of success in Table 5. In this section, "inspected" refers to determining whether the component is functioning or not, and "repairing or replacing" refers to returning that component back to normal operating behaviour.

The first case is when the components are diagnosed correctly: these are the combinations $6,7,8,9,15$ and 28 . As can be seen in Table 5, the BBN confirms that these combinations of components have failed and it diagnoses the failures correctly. Therefore, the system can return to normal operating conditions by inspecting and addressing (repairing or replacing) the same number of components, as the number of components that have failed.

The second case is when the components are diagnosed correctly, but the BBN identifies the component failures with the highest (but not 100\%) probability of failure. These cases are combination 5 (with 95.84\% probability) and combinations 10 and 14 (with $95.47 \%$ probability for one of the components and $100 \%$ for the other component). For combinations 10 and 14 the same set of sensor readings in each case can be produced by other combinations of 3 or more component failures. For example, the readings for 
combination 10 can be also produced by a combination of component failures, P1, V1 and V3. Hence for combinations 10 and 14 the probability for the second component failure in less than $100 \%$. Therefore, the BBN also diagnoses the failures for these combinations correctly, and the system can return to normal operating conditions by inspecting the same number of components as the number of components that have failed, as in the first case.

The third case is when the failures are not guaranteed to be diagnosed correctly at the first attempt. This is because two different component failures produce the same symptoms. The combinations of component failures are 1, 2, 3 and 4 . There is a $50 \%$ probability that a component failure will be diagnosed correctly, as both failures are equally likely to occur. This means that the component failure will be diagnosed correctly if both failures can be inspected, i.e. $50 \%$ of the time, there will be one component that is inspected but it has not failed. Therefore, the system will be returned to normal operating conditions by inspecting one or two components, when there is one component failure.

The fourth case is similar to the third case, when an additional component to the component failures, described in the third case, is failed. These combinations are 12, 13, $17,18,21,22,24$ and 25 . In this case, the system will be returned to normal operating conditions by inspecting up to three components, when there are two component failures.

The fifth case is when the diagnosed component failure is valve V3, but the actual failure involves another failure, valve V4 or valve V5, as in combinations 26 and 27, where the symptoms are the same. Therefore, as it is significantly more likely that only one component has failed than two components have failed, the BBN outputs that only valve V3 has failed. However, if valve V3 is repaired, the sensors will produce readings that are not the same as those obtained during the normal operating conditions. When this evidence is introduced to the BBN, the correct component failure will then be diagnosed. Therefore, the system will be returned to normal operating conditions by inspecting two components when there are two component failures.

The penultimate case, the sixth case, is similar to the fifth case: when valve V3 is repaired it will become apparent that there is another failure. This will then result in the situation discussed in the third case. This case is different to the fourth case because in the fourth case the results of the BBN suggest that there are two failures straight away, but the sixth case requires valve V3 to be repaired/replaced before it detects that there is a second failure. These combinations are 11,16, 20 and 23. Therefore, the system will be returned to normal operating conditions by inspecting either two or three components, when there are two component failures.

The final case, the seventh case, is when the $\mathrm{BBN}$ predicts that valve $\mathrm{V} 3$ is failed with probability of $95.84 \%$. However, using these sensors, valve V1 and valve V2 failure produces the same sensor readings, combination number 19. As this combination is significantly less likely to occur than valve V3 failing, it is not diagnosed correctly. When this component is inspected and found to be not failed, this evidence can be introduced to the BBN. This will then update the probability that each of the other components are in their failed state and will then diagnose the failures correctly. Therefore, the system will be returned to normal operating conditions by inspecting three components, when there are two component failures.

The example cases discussed above result in the diagnostic term being less than $1,\left(D I_{\{s\}}\right.$ $=0.6818$ ), as there are some cases where failures cannot be diagnosed correctly. However, the reason that these cases were not diagnosed correctly is because the symptoms produced 
are exactly the same for multiple different component failures. The performance could not be improved by including more sensors.

If the best combination of four sensors is considered, the final case, (combination 19), would be diagnosed correctly on the first attempt. This is because introducing either sensor $\mathrm{S} 2$ or S4 will give the additional information required to diagnose this failure combination correctly, and diagnosing this failure correctly is the only benefit of adding the fourth sensor to the combination.

In the next section, an analysis of the methodology is given, along with some potential improvements.

Table 5 Diagnosis of component failures using S1, S3 and S7

\begin{tabular}{|c|c|c|c|c|c|c|c|c|}
\hline Case & No. & $\begin{array}{l}\text { Actual } \\
\text { failure }\end{array}$ & $\begin{array}{c}\text { Diagnosed } \\
\text { Failure } 1\end{array}$ & Probability & $\begin{array}{l}\text { Diagnosed } \\
\text { Failure } 2\end{array}$ & Probability & $\begin{array}{c}\text { Diagnosed } \\
\text { Failure } 3\end{array}$ & Probability \\
\hline \multirow[t]{6}{*}{1} & 6 & V4 & V4 & $100 \%$ & & & & \\
\hline & 7 & V5 & V5 & $100 \%$ & & & & \\
\hline & 8 & P1 P2 & P1 & $100 \%$ & P2 & $100 \%$ & & \\
\hline & 9 & P1 V1 & P1 & $100 \%$ & V1 & $100 \%$ & & \\
\hline & 15 & P2 V2 & P2 & $100 \%$ & V2 & $100 \%$ & & \\
\hline & 28 & V4 V5 & V4 & $100 \%$ & V5 & $100 \%$ & & \\
\hline \multirow[t]{3}{*}{2} & 5 & V3 & V3 & $95.84 \%$ & & & & \\
\hline & 10 & $\mathrm{P} 1 \mathrm{~V} 2$ & $\mathrm{P} 1$ & $100 \%$ & V2 & $95.47 \%$ & & \\
\hline & 14 & P2 V1 & $\mathrm{P} 2$ & $100 \%$ & V1 & $95.47 \%$ & & \\
\hline \multirow[t]{4}{*}{3} & 1 & P1 & P1 & $50 \%$ & V1 & $50 \%$ & & \\
\hline & 2 & $\mathrm{P} 2$ & $\mathrm{P} 2$ & $50 \%$ & V2 & $50 \%$ & & \\
\hline & 3 & V1 & P1 & $50 \%$ & V1 & $50 \%$ & & \\
\hline & 4 & V2 & $\mathrm{P} 2$ & $50 \%$ & V2 & $50 \%$ & & \\
\hline \multirow[t]{8}{*}{4} & 12 & P1 V4 & V4 & $100 \%$ & $\mathrm{P} 1$ & $50 \%$ & V1 & $50 \%$ \\
\hline & 13 & P1 V5 & V5 & $100 \%$ & $\mathrm{P} 1$ & $50 \%$ & V1 & $50 \%$ \\
\hline & 17 & P2 V4 & V4 & $100 \%$ & $\mathrm{P} 2$ & $50 \%$ & V2 & $50 \%$ \\
\hline & 18 & P2 V5 & V5 & $100 \%$ & $\mathrm{P} 2$ & $50 \%$ & $\mathrm{~V} 2$ & $50 \%$ \\
\hline & 21 & V1 V4 & V4 & $100 \%$ & P1 & $50 \%$ & V1 & $50 \%$ \\
\hline & 22 & V1 V5 & V5 & $100 \%$ & $\mathrm{P} 1$ & $50 \%$ & V1 & $50 \%$ \\
\hline & 24 & V2 V4 & V4 & $100 \%$ & $\mathrm{P} 2$ & $50 \%$ & V2 & $50 \%$ \\
\hline & 25 & $\mathrm{~V} 2 \mathrm{~V} 5$ & V5 & $100 \%$ & $\mathrm{P} 2$ & $50 \%$ & $\mathrm{~V} 2$ & $50 \%$ \\
\hline \multirow[t]{2}{*}{5} & 26 & V3 V4 & V3 & $95.84 \%$ & & & & \\
\hline & 27 & V3 V5 & V3 & $95.84 \%$ & & & & \\
\hline \multirow[t]{4}{*}{6} & 11 & P1 V3 & V3 & $95.84 \%$ & & & & \\
\hline & 16 & $\mathrm{P} 2 \mathrm{~V} 3$ & V3 & $95.84 \%$ & & & & \\
\hline & 20 & V1 V3 & V3 & $95.84 \%$ & & & & \\
\hline & 23 & $\mathrm{~V} 2 \mathrm{~V} 3$ & $\mathrm{~V} 3$ & $95.84 \%$ & & & & \\
\hline 7 & 19 & V1 V2 & V3 & $95.84 \%$ & & & & \\
\hline
\end{tabular}

\section{Analysis of the proposed methodology}

The first step of the methodology is to calculate the performance metric of individual sensors and combinations of sensors. The maximum possible performance metric can be 
calculated by considering all of the sensors on the system. This can give the analyst a target to achieve, and can, therefore, reduce the risk of wasting resources attempting to achieve a better performance metric exhaustively. For example, for this example system, combinations of 5 sensors could have been calculated unnecessarily.

The proposed methodology could be modified, since it was observed that the BBN model could be used to calculate the sensor performance metric automatically. Component state evidence can be introduced to the network, and the BBN will automatically produce the sensor readings and the probability of occurrence of the evidence that has been introduced. This means that if this is repeated for all possible component failure combinations, the three terms of the performance metric can be calculated, and therefore, the performance metric can also be calculated. This would be particularly useful for complex systems with a large number of components and sensors.

\subsection{Sensor selection}

In section 3.1.5, it was suggested that the performance metric, given in Equation 12, should only be used as a guide to narrow down the selection of sensors. The higher ranked combinations should then be studied in more detail, looking at each of the terms individually in order to select the sensors to be used on the system. This will result in the analyst having the ability to select the combination of sensors that has certain terms higher than others, if desired. This would eliminate the risk of selecting a sensor combination with one term that is significantly lower than desired, making the sensor selection more applicable.

\subsection{Modelling the system}

The system modelling technique of constructing a BBN of the system produces a good representation of the example system. The components and sensors in the system are represented using nodes in the BBN model and this allows the relationship between the component states and sensor readings to be modelled consistently. The sensor readings that would be output by a real system are output by the BBN model.

The example system is a simple system, for example, partial failures, leaks, pipe blockages etc. are not considered, which reduces the accuracy of the model. The BBN model could be constructed considering the additional failure modes, which would increase its size. If BBNs could not be scaled up to large systems, alternative system modelling techniques will have to be considered.

\subsection{Fault diagnostics}

In section 4.3, the fault diagnostic process was applied to the system. It was shown that it successfully diagnosed all of the component failures. In some cases, the diagnosis was not completely correct initially, but it was always able to diagnose the failures whilst inspecting no more than one component that has not actually failed. However, the accuracy of the diagnostics process could be improved slightly by considering one of the combinations of 4 sensors, presented in section 4.1.1. As discussed in section 4.3, using four sensors would result in failure number 19 (case number 7 in Table 5) to be diagnosed correctly, i.e. $100 \%$. No further improvement could be achieved even by using all 11 sensors, i.e. there would be still the same cases where the diagnostics is not completely correct. This is because some component failures produce the same set of symptoms and 
are, therefore, indistinguishable, for example, with the current set of sensors failures of pump P1 and valve V1 produce the same set of symptoms.

One of the key benefits of the BBN methodology is that it outputs the probabilities of events that each of the components have failed. Therefore, a certain threshold could be set and a spare for any component that has a probability of being failed above it could be taken to the aircraft, when it is attended to by the maintenance team. This would reduce the likelihood of taking a large number of replacement components unnecessarily, whilst also reducing the time waiting for other components to be brought to the system, if the most likely failure has not occurred. This could happen when there are multiple components with a similar probability of failing. In the example system given, if the sensor reading combination produced is combination 1, then both replacement components for pump P1 and valve V1 would be taken to the system. Doing this would achieve a reduction in the overall downtime of the system, reducing waiting time for replacement components that have been misdiagnosed.

\subsection{Fault diagnostics using an alternative sensor selection method}

The performance of the selected sensors using the proposed method and an alternative method is briefly compared in terms of fault diagnostics results. Using the fault symptom matrix approach by Snooke ${ }^{1}$ (presented in Introduction) any of the combinations of three sensors given in Table 3 could have been chosen as the best, as any of these combinations can detect all component failures. For example, if the combination of sensors S2, S4 and $\mathrm{S} 8$ is chosen, the diagnostics results are given in Table 6.

Table 6 Diagnosis of the component failures using S2, S4 and S8

\begin{tabular}{lllllllll}
\hline Case & No. & $\begin{array}{c}\text { Actual } \\
\text { failure }\end{array}$ & $\begin{array}{c}\text { Diagnosed } \\
\text { Failure 1 }\end{array}$ & Probability & $\begin{array}{c}\text { Diagnosed } \\
\text { Failure 2 }\end{array}$ & Probability & $\begin{array}{c}\text { Diagnosed } \\
\text { Failure 3 }\end{array}$ & Probability \\
\hline 1 & 6 & V4 & V4 & $100 \%$ & & & & \\
& 7 & V5 & V5 & $100 \%$ & & & & \\
\hline 2 & 5 & V3 & V3 & $95.47 \%$ & & & & \\
\hline 3 & 1 & P1 & P1 & $51.28 \%$ & V1 & $51.28 \%$ & & \\
& 2 & P2 & P2 & $51.28 \%$ & V2 & $51.28 \%$ & & \\
& 3 & V1 & P1 & $51.28 \%$ & V1 & $51.28 \%$ & & \\
& 4 & V2 & P2 & $51.28 \%$ & V2 & $51.28 \%$ & & $51.28 \%$ \\
\hline 4 & 12 & P1 V4 & V4 & $100 \%$ & P1 & $51.28 \%$ & V1 & \\
& 13 & P1 V5 & V5 & $100 \%$ & P1 & $51.28 \%$ & V1 & $51.28 \%$ \\
& 17 & P2 V4 & V4 & $100 \%$ & P2 & $51.28 \%$ & V2 & $51.28 \%$ \\
& 18 & P2 V5 & V5 & $100 \%$ & P2 & $51.28 \%$ & V2 & $51.28 \%$ \\
& 21 & V1 V4 & V4 & $100 \%$ & P1 & $51.28 \%$ & V1 & $51.28 \%$ \\
& 22 & V1 V5 & V5 & $100 \%$ & P1 & $51.28 \%$ & V1 & $51.28 \%$ \\
\hline & 24 & V2 V4 & V4 & $100 \%$ & P2 & $51.28 \%$ & V2 & $51.28 \%$ \\
& 25 & V2 V5 & V5 & $100 \%$ & P2 & $51.28 \%$ & V2 & $51.28 \%$ \\
& 9 & P1 V1 & P1 & $51.28 \%$ & V1 & $51.28 \%$ & & \\
& 15 & P2 V2 & P2 & $51.28 \%$ & V2 & $51.28 \%$ & & \\
& 8 & P1 P2 & P1 & $51.28 \%$ & P2 & $51.28 \%$ & V1 & $51.28 \%$ \\
& 10 & P1 V2 & P1 & $51.28 \%$ & P2 & $51.28 \%$ & V1 & $51.28 \%$ \\
& 14 & P2 V1 & P1 & $51.28 \%$ & P2 & $51.28 \%$ & V1 & $51.28 \%$ \\
& & & & & & & &
\end{tabular}




\begin{tabular}{lllllllll} 
& 19 & V1 V2 & P1 & $51.28 \%$ & P2 & $51.28 \%$ & V1 & $51.28 \%$ \\
\hline 6 & 26 & V3 V4 & V3 & $95.47 \%$ & & & & \\
& 27 & V3 V5 & V3 & $95.47 \%$ & & & & \\
\hline 7 & 11 & P1 V3 & V3 & $95.47 \%$ & & & & \\
& 16 & P2 V3 & V3 & $95.47 \%$ & & & & \\
& 20 & V1 V3 & V3 & $95.47 \%$ & & & & \\
& 23 & V2 V3 & V3 & $95.47 \%$ & & & & \\
\hline 8 & 28 & V4 V5 & V3 & $95.47 \%$ & & & \\
\hline
\end{tabular}

By comparing Tables 5 and 6 , it can be observed that the diagnostics results using S2, S4 and S8 are slightly worse, for example, there are fewer combinations of component failures that are diagnosed correctly with $100 \%$ probability (case 1), i.e. 2 failures in Table 6 instead of 6 failures in Table 5. A similar situation is with case 2, as there are fewer combinations that can be diagnosed correctly. Note, that the result for component failure 19 (which could not be diagnosed correctly in Table 5) has now improved as one of the component failures, V1, is identified correctly, however, there is another failure (failure 28 ) in Table 6 that cannot be diagnosed correctly. Overall, it can be said that the proposed method can be used to identify a suitable set of sensors, especially in situations when the diagnostics capability is of importance.

\section{Conclusions and future work}

In summary, this paper proposed a methodology for selecting sensors and using the sensors to diagnose failures that may occur in the system. The sensor selection method entails using a newly developed performance metric, which considers the sensor ability to detect faults and diagnose failures, as well as considering the effects of the detected failures on the system performance. It was suggested that after considering the average of the three terms for the performance metric, the three terms of the performance metric should then be considered individually for the final sensor selection process. This will enable the analyst to consider each of the three terms individually and select the sensors that best suit the considered application.

A BBN-based system modelling methodology was developed using the selected sensors. A BBN is constructed for the system which includes nodes for the components, sensors and other nodes, as required. Sensor readings can be introduced to the BBN in the form of evidence and they are used to update the probability of each of the component states. This process enables the component failures to be diagnosed, and it can be used to restore system operation as quickly as possible.

There are a number of potential future research areas that could be explored. The first of these is to analyse the scalability of the methodology, by testing it on a larger system. It may require some automation of the sensor selection process, particularly the calculation of the sensor performance metric. It would also be beneficial to consider multiple system operation phases. This would enable phased missions to be modelled, as it is observed on aircraft systems, such as take-off, cruise, fuel transfer and landing. As different components will have different behaviour during the mission phases, more component failure modes will need to be considered. When the complexity of the system increases, an optimisation technique, such as Genetic Algorithms, could be applied together with the sensor selection process. In that case the solution would be obtained without the need to 
evaluate a large number of sensor combinations exhaustively. The complexity of the considered sensors could also be increased by considering the sensors cost and sensor failure modes in the proposed method.

\section{Acknowledgments}

This project is supported by BAE Systems and the Engineering and Physical Sciences Research Council (EPSRC). The authors gratefully acknowledge the support of these organisations.

\section{References}

1. Snooke N. An automated failure modes and effects analysis based visual matrix approach to sensor selection and diagnosability assessment. In: IEEE conference on Prognostics and Health Management (PHM). 2009.

2. Kang CW and Golay MW. An integrated method for comprehensive sensor network development in complex power plant systems. Reliability Engineering \& System Safety, 2000; 67(1): 17-27.

3. Memik SO, Mukherjee R, Ni M. and Long J. Optimizing thermal sensor allocation for microprocessors. IEEE Transactions on Computer-Aided Design of Integrated Circuits and Systems, 2008; 27(3), pp.516-527.

4. Mukherjee R, Mondal S and Memik SO. Thermal sensor allocation and placement for reconfigurable systems. In: Computer-Aided Design, 2006. ICCAD'06. IEEE/ACM International Conference on (pp. 437-442). IEEE. 2006.

5. Maul W, Kopasakis G, Santi L, et al. Sensor Selection and Optimization for Health Assessment of Aerospace Systems. Journal of Aerospace Computing, Information, and Communication 2008; 5(1): 16-34.

6. Khan A and Ceglarek D. Sensor optimization for fault diagnosis in multi-fixture assembly systems with distributed sensing. Journal of manufacturing science and engineering. 2000; 122(1), pp.215-226.

7. Pourali M and Mosleh A. A Bayesian approach to sensor placement optimization and system reliability monitoring. Proceedings of the Institution of Mechanical Engineers, Part O: Journal of Risk and Reliability 2013; 227(3): 327-347.

8. Lambert JH, and Farrington MW. Cost-benefit functions for the allocation of security sensors for air contaminants. Reliability Engineering \& System Safety, 2007; 92(7): 930-946.

9. Santi LM, Sowers TS, and Aguilar RB. Optimal sensor selection for health monitoring systems, National Aeronautics and Space Administration, 2005; 19(1): 39-52.

10. Spanache S, Escobet T and Travé-Massuyes L. Sensor placement optimization using genetic algorithms. In: $15^{\text {th }}$ international Workshop on Principles of Diagnosis (DX'04), 23 June 2004, 179-184.

11. Rosich A, Frisk E, Aslund J, Sarrate R, and Nejjari F. Sensor Placement for Fault Diagnosis Based On Causal Computations. In: 7th IFAC Symposium on Fault Detection, Supervision and Safety of Technical Processes, SAFEPROCESSO9, Barcelona, Spain, 2009, pp. 402-407. 
12. Sarrate Estruch R, Nejjari Akhi-Elarab F and Rosich Oliva A. Sensor placement for fault diagnosis performance maximization under budgetary constraints. In: Proceeding of the International Conference on Systems and Control. 2012.

13. Krysander M and Frisk E. Sensor placement for fault diagnosis. IEEE Transactions on Systems, Man, and Cybernetics-Part A: Systems and Humans 38.6. 2008: 1398-1410.

14. Hurdle EE, Bartlett LM and Andrews JD. System fault diagnostics using fault tree analysis. Proceedings of the Institution of Mechanical Engineers, Part O: Journal of Risk and Reliability 2008; 221 (1): 43-55.

15. Hurdle EE, Bartlett LM and Andrews JD. Fault tree based fault diagnostics methodology for an aircraft fuel system. In: Proceedings of the 32nd ESREDA Seminar: Maintenance Modelling and Applications, Algerho, Italy, 8 May - 9 May 2007.

16. Bartlett LM, Hurdle EE and Kelly EM. Comparison of digraph and fault tree based approaches for system fault diagnostics. In: Proceedings of the European Safety and Reliability Conference, Estoril, Portugal, 18 September - 22 September 2006. 1: 191-198.

17. Lloyd MD, Andrews JD, Remenyte-Prescott R, Pearson JT and Hubbard Peter. A Petri Net Approach to Fault Verification in Phased Mission Systems using the Standard Deviation Technique. Quality and Reliability Engineering International 2014; 30(1): 83-95.

18. Lampis M and Andrews J. Bayesian belief networks for system fault diagnostics. Quality and Reliability Engineering International 2009; 25(4): 409-426.

19. Cheok MC, Parry GW and Sherry RR. Use of importance measures in riskinformed regulatory applications. Reliability Engineering \& System Safety 1998; 60(3): 213-226.

20. Koski T and Noble JM. Bayesian Networks: An Introduction. $1^{\text {st }}$ ed. John Wiley \& Sons LTD, 2009.

21. Mittal S and Maskara SL. A review of some Bayesian Belief Network structure learning algorithms. In: Information, Communications and Signal Processing (ICICS) 2011 8th International Conference on (pp. 1-5). IEEE. 